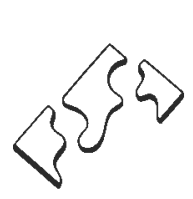

\title{
"There's a real dog in the classroom": The Relationship Between Young Children and Animals
}

Marjan Margadant-van Arcken

University of Utrecht

In this article I will describe the results of a research project on the relationship between four- to six-year-old children and (pet) animals.

I visited two kindergartens: one in the old harbor district of Rotterdam and another in a village in the south of the Netherlands. In the Rotterdam kindergarten, all the children are from working class families. Almost half of the school population has various ethnic backgrounds; the children come from Surinam, Chinese, Portuguese, Moroccan and Yugoslav families.

In the village kindergarten, the children have a wide range of socialeconomic backgrounds: they vary from gypsy children to the children of dentists and physicians.

I visited each school for three weeks and each day I brought a different pet with me. I used my own Cairn terriers, which are very small dogs, my short-hair British-Blue cats and a guinea pig.

Each day I started with a group conversation. The children were encouraged to react spontaneously, to ask questions or to talk about their own experiences with animals. These conversations were tape recorded.

After these conversations, the children were allowed to play with the animals in small groups. I made short notes on these free-play situations, and these notes were transcribed extensively in a research journal the same day.

Also, I visited a children's farm with the children of the Rotterdam kindergarten.

\section{The Development of the Relationship Between the Child and the Animal}

During the project, it became clear that the meeting of a child and a strange animal follows a more or less structured pattern. The child's basic attitude is a little fearful. He approaches the animal carefully, 
takes a short look at it and after that he strokes the animal's back. He never strokes the animal's head immediately.

This basic attitude remains during the phases of meeting and developing a relationship with the animal. Only when the child is completely at ease with the animal does he lose his fear.

Immediately after the first careful stroke along the back of a strange animal, the same question follows: "What's his name?" This is an essential question for children, as they approach the animal as an individual and not as a nameless representative of the species.

Young children always select meaningful or cuddly names for animals. Adults often select beautiful, aesthetic names, but children change these names to their own taste when playing with the animal.

In the doll's corner, the children are playing vet with the tom-cat Bas.

Daniëlle walks around with Bas and says: "We call him Katja."

A child gets to know a strange animal by doing something with it. But what you can do with an animal depends to a large extent on the nature of the animal itself. The play activities of young children and animals therefore show considerable variety, such as walking around with the animal, showing the school to the animal and showing the animal to other people, feeding it, grooming it, playing with its toys, etc.

During the development of the relationship, the fear of an animal can break through at any moment. This is not necessarily always real fear; it can range from mild fright to fear to real panic.

Marcel and Jurman are running down the corridor. Toek, the dog, is running along with them and Marcel releases the leash. Toek barks and jumps up against Jurman; the dog is very fond of wild games, but Jurman screams in full fear: "He bites, he bites."

Because he is unfamiliar with the behavior of dogs, Jurman makes the wrong interpretation. Young children often make these mistakes.

To master their vague fear of animals, young children play "let's pretend" games. They pretend that the animal is very dangerous.

Hassan and Yan-Fook are playing with the cat. They pretend they are very afraid of him. They stalk the cat, flee up the jungle-gym and laugh with great pleasure.

The time required for developing a trust relationship with an animal differs not only from child to child, but also from animal to animal. It depends heavily on the size and the behavior of the animal. The 
guinea pig, for example, is relatively small and most of the time it is in its cage. The guinea pig is accustomed to children and not at all shy. It does not show unpredictable behavior. It was allowed to walk around freely during the group conversation. And every child could hold it for a short while. Therefore, the children learned very quickly what they can and cannot expect of this animal. This takes more time with the dog and even more time with the cat, as the behavior of the cat is much more unpredictable.

As soon as a trust relationship with the animal has been established, the animal may share in the normal activities of the children. The animal becomes part of their game when they are playing in the doll's corner or building a tent. Also, the children dig holes in the sandtable or the sandbox and build houses of bricks for the animal. They often make these holes and houses too small. As soon as they try to fit in the animal, they see their mistake and say: "He looks like a giant," but they immediately make new, bigger ones and are very happy when the animal fits in and appears to enjoy sitting there.

By playing in this way, the child and the animal share one reality. A "fusion of horizons" has been established, based on the symbiotic understanding of a mutual experience.

Children also imitate the behavior of animals:

Some children are digging in the sandbox. The dog sees this and also starts to dig. The children laugh about this and start digging again, but now like dogs.

For the dog, this sharing has its limits. If the children were to dig with a spade, it would be impossible for the dog to imitate this.

\section{The Animal Image of Young Children}

Young children have a very direct and exact perception of things. They immediately see small differences between animals of the same species.

The two British-Blue cats look very much alike. At home we often have problems in seeing who's who. But the children immediately see differences: "That one looks angry and the other one's tail is shorter."

The perception is not only very direct, but also very intense. The child looks at the animal with all his attention.

In the summer, my youngest son was looking at the butterflies. In the middle of a host of butterflies, there was an Atalanta with vibrating wings. I stood behind my son and saw his shoulder-blades move. Then I realized that at that moment he was a butterfly with the other butterflies. 
Touching the animal is also a very active and primary form of perception. Young children look with their hands and enjoy very intensely the softness of an animal.

For children who own rabbits, all the other animals are "hard." They brush the guinea pig, for example, until he is "soft." On the children's farm there are, besides many other animals, grasshoppers and blind-worms. The children are very afraid of them, but they also don't dare to touch them, because they think that will be a disagreeable feeling.

A young child's experience of his environment is influenced considerably by his own desires and feelings. When a child gives an explanation for the meaning of animal behavior-why an animal does something-he sees it from his own perspective.

The tom-cat Bas has the habit of letting a little piece of his tongue hang out of his mouth. "Doesn't he like us?", a little girl asks and all the children stick out their tongues at Bas.

\section{Another example:}

At the children's farm, a teacher asks: "Why do animals walk around?" "To see other children," Fatima says. That's the wrong answer. "To see other animals," Jurman tries. Wrong again. No-one knows the correct answer. "To find food," the teacher says at last. The children don't react; the answer lies beyond their horizon.

In the same way we must understand why children enjoy teasing animals. A little child pinches an animal or pulls its tail to provoke the animal to react. He does not want to hurt it, but likes to make the animal move. Adults also have the inclination to knock against an aquarium to make the fishes swim.

Therefore, it is important for children that they can do something with an animal. Also, it seems important that the animal moves in a predictable way. Unpredictable movements are scary. Most of the children in this project favored rabbits: "Because he is so soft"; "Because he doesn't move"; "Because he's mine." This shows that having an animal of your own is important as well. On being asked: "Which animal do you like most?", Michiel replies: "My Johnny." Johnny proves to be his rabbit.

Next to that, young children favor small animals. They do not distinguish between small and young. The smallness of the animal is important to them and its youthfulness (playfulness) is an extra attractive quality.

Small animals are easier to handle for little children. This is due to their motoric development. At this age the lateralisation process has 
not yet been completed. They cannot hop because they cannot use their feet alternately. Their motoric organization is still exclusively symmetric. When young children pick up an animal, they do that with two hands on each side of the animal's back: left hand and right hand make the same movement. That is the reason why young children pick up an animal in such a clumsy way. They simply cannot do it the way we do.

This motoric development partly influences the child's fear of animals. When you are not that handy, it is even more difficult to hold a wriggly guinea pig.

In children's drawings, you can see clearly that the animal image of young children is a situational one and not an integrated totality. Like little cartoonists they draw different situations on one sheet of paper, using them to show the course and the variation of their experiences.

Patricia wants to make me a drawing of the dog. But she doesn't draw one dog, as an abstraction of all her experiences, no, she draws on one sheet of paper, herself with the dog on the leash, the dog in the sandbox, and the dog running around freely with the ball.

Certainly she knows that the dog on the leash, the dog in the sandbox and the dog running around freely are one and the same. But she is indicating that her animal image consists of little pieces.

\section{Contrast}

There are considerable contrasts between young children. In play situations the differences between four-, five-, and six-year-old children are easy to see. Four-year-olds don't play with the animal. They drag the animal with them in the direction they want to go. When playing with the ball and with the dog, the dog is not allowed to pick up the ball himself. He is pulled back with the leash and another child picks up the ball.

It struck me that, in the course of only one year, a child learns to take the animal into consideration, to make way so that the animal can play as well.

Five- or six-year-old children will always ask whether the animal is male or female. It is great fun to return that question: "What do you think?" The answers show that Levinson's (1972) statement is correct: "Animals are usually seen as males rather than females" (p. 18). This is strongly anchored in our daily (Dutch) language use. An animal is a "he"; Young children don't hear anything else and this does not encourage their thinking about animals from a different sex perspective. 
Only a small number of children like to take care of an animal. All children like to feed an animal, but really taking care of one is exceptional. In this project with 150 children, only one five-year-old girl really took care of the dog during the whole morning.

I did not see many differences between boys and girls concerning animals. Boys often treat animals like cars.

The guinea pig is in the cage. A boy seizes it by its back, says: "It's like a car" and pushes the guinea pig back and forth like a car.

Girls like to dress up the animals with ribbons and clothes. They also decorate their animal drawings with all kinds of things.

There are important differences between children from ethnic minorities and Dutch children. Children from ethnic minorities have the problem of living in two cultures: The native culture at home and the Dutch culture at school.

We know little about the animal image in other cultures. But keeping a pet is an almost unknown phenomenon in countries with a low standard of living.

The children of immigrants in this research project have no animals at home, so they cannot learn how to form an affective relationship with animals at home. For them it is even more strange to see a real live animal in the classroom and it takes a while for them to get used to it. Also, they are more fearful than the Dutch children.

Among the teachers, the prejudice is common that "foreign" children are cruel to animals. But this apparent cruelty proves to be a different view on animals.

Hassan (a Moroccan child) and Yan-Fook (a Chinese child) are walking with Bas in the corridor, where some water has been spilt. "Be careful, slippery," Hassan says to Bas. They go into the kitchen to give the cat some milk. There are knives lying on the dresser. "Cut dead to eat," Hassan says and Yan-Fook pretends to cut the cat in half. Together they "eat" the cat.

Afterwards, the teachers say: "We told you so." However, "slaughtering" the cat in this way is not cruelty; these children are only showing that for them an animal, even a pet, can mean food.

We have to be very careful in our interpretation of the behavior of foreign children. We do not know exactly what problems they will have if we urge them too strongly to play with animals.

Village children have more animals at home than city children, so that they know more about animals and have had more experiences 
with animals. They are acquainted with the life cycle of an animal: birth-life-death. Mothers bring all kinds of young animals to school, even little lambs and goats in big shopping bags.

Usually the relationship of village children with production animals is not as close as their relationship with domestic pets, but young children often develop a relationship with a certain calf: "It's so nice when he licks me," Lotje says.

Also, the animal's death is part of their experience. In Rotterdam, on the contrary, the death of a pet is consciously hidden from young children. Village children, however, bury their pets in the garden, often together with their parents.

José says: "Our Daddy drove over our puppy with the car, because he never looks out properly, our puppy, he's buried now. Then Ingrid and Mark came, they came and haunted us."

In one and the same breath, the experience of death is followed by a ghost story. In my opinion, this shows that the death experience is also frightening for young children. The shock of the sudden death of the dog is acted out by playing ghost.

During the research project, the children made my animals disappear by magic. If I then started to "cry," they quickly made the animals reappear. They played this game with me many times. They tried me out to see how I would react to the loss of a beloved animal. In this way they acted out the power of magic and feelings of sorrow in a "let's pretend" game.

\section{Conclusion}

We can construe grand theories to show how important animals are for the child's affective and emotional development; how the animal can be a medium between the child and reality; how many educational values can be transferred by means of animals, e.g., learning to shoulder responsibilities. But that, I am convinced, does not bring us to the core of the matter.

Young children are not capable of expressing in words what animals mean to them. A few times I heard a child say that he likes taking the cat to bed. Why is that so nice? Because the child is no longer so alone in bed. The cat shares his loneliness and in that way the loneliness is easier to endure.

In my view, the "fusion of horizons," the shared life space, is the core of the relationship between the child and the animal. It is based on a mutual, symbiotic understanding, but it is the child who gives the contact meaning. 


\section{Notes}

1. This research project will be extended to three more schools, including a school for handicapped children. Moreover, a historical study is being made of the relationship between children and animals during the period 1850-1980.

2. Those who wish to submit remarks, advice, research results or literature related to the subject, are cordially requested to write to the author at: Amethistdijk 236, 4706 BH Roosendaal, The Netherlands.

3. There is a report available to Dutch readers: "Er zit een échte hond in de klas."

\section{References}

Levinson, B. M. (1972). Pets and Human Development. Illinois. 